\title{
El talante como categoría moral
}

\author{
CARLOS THIEBAUT
}

José Luis López Aranguren acuñó y empleó filosóficamente el término talante en dos de sus obras centrales de mediados de los años cincuenta, Catolicismo y protestantismo como formas de existencia y Ética. En estas obras la idea de talante juega de manera distinta e, incluso, podríamos sugerir que pertenece a mundos conceptuales distintos cuya tensión permitirá, posteriormente, nuevas interpretaciones del concepto en las que lo anteriormente dicho se mantiene aunque matizado. En efecto, en los años setenta y ochenta Aranguren regresa al análisis de nuestro concepto y en El buen talante recupera parte del primero de esos textos en una nueva edición a la que no le faltan modificaciones ${ }^{1}$. En Catolicismo y protestantismo... la idea de talante constituye una categoría moral que está más bien diseñada para el análisis de las formas de la personalidad religiosa, y en concreto, de las formas de la religiosidad de la modernidad, un análisis al que Aranguren dedicaría unas energías de autoexploración propia nada pequeñas en aquella década. Pero también esa obra de 1952 comienza a explorar algunas dimensiones sociales de la idea de talante; por ello, también es en parte una categoría de la sociología de la moral que enlaza con otros intereses arangurenianos desarrollados en los años sesenta y que aquí no tocaremos. El talante es, por tanto, una categoría puente entre las (distintas) maneras de ser del hombre y las (distintas) circunstancias históricas en las que vive. En la Ética, de 1958, y tal vez dado el carácter más académico y sistemático del libro, esa idea pertenece sobre todo a lo que cabría denominar la analítica de la moral y de la virtud. Por emplear también términos arangurenianos - que proceden de tradiciones anteriores que no es el momento de analizar- cabría sugerir que en Catolicismo y protestantismo... la idea de talante pertenece al ámbito de la moral vivida, de la ética utens, aquella que se afana en autocomprendernos en nuestro vivir, mientras que en la Ética se ubica en el análisis teórico de lo moral, de la ética docens, aquella que sistematiza y categoriza para enseñar la estructura de nuestra moralidad. La idea de talante, pues, es una idea en tensión, un concepto de semántica o de usos múltiples aunque conserve siempre un cierto aire de familia. Su pertenencia, o su juego, en esos contextos filosóficos de diferentes intencionalidades, estructura e, incluso, influencias e intereses, permite una fuerte versatilidad en su uso a la vez que induce algunos problemas de comprensión a cuyo esbozo y clarificación quisieran contribuir estas líneas tratando de acen-

' Citaremos por sus Obras Completas, vols. I y II, ed. de F. Blázquez, Madrid, Trotta, 1994. Se referirá $O C$, vol. y p. Catolicismo y protestantismo... se recoge en $O C, \mathrm{I}, 211-411$; Ética en $O C, \mathrm{II}, 161-502 ;$ El buen talante en $O C, \mathrm{II}, 619-671$. 
tuar ese parecido de familia al que hemos aludido. Analizaremos primero el concepto de talante en Catolicismo y protestantismo..., una obra que, según se nos indica en el Prólogo de 1980, había comenzado a elaborarse ya en $1944^{2}$. Indicaremos, al hilo de ese análisis, y aunque sea brevemente, las modificaciones que la introducción a Catolicismo..., bajo la rúbrica de El talante religioso, sufre al volver a presentarse en 1985. En segundo lugar, mostraremos las scmejanzas y, sobre todo, las diferencias del tratamicnto de ese concepto en la Etica. Concluiremos con una sugerencia para su comprensión actual: tal vez la noción de talante, convenientemente matizada o modificada, pueda ser una buena manera de traducir al lenguaje filosófico castellano algunos desarrollos contemporáneos sobre la sensibilidad moral, desarrollos con los que parte de la más reciente ética analítica ha querido recuperar la idea clásica de virtud moral.

\section{El talante entre la cultura y la existencia}

Si el concepto de talante es un concepto en tensión en los dos contextos y obras de los cincuenta que hemos indicado, podemos también apuntar que el libro mismo Catolicismo y protestantismo... muestra una peculiar y propia tensión interna en la que ese concepto tiene un especial papel. El lector contemporáneo (lejano, quizá, del contexto cultural de aquellos años oscuros) no deja de percibir que la manera como el libro arranca - en su primera aplicación de la teoría del talante- difiere sensiblemente del tono con el que concluye. Las páginas iniciales parecen apuntar a una tesis, o a una hipótesis, según la cual mientras el protestantismo primaría un peculiar talante religioso de tono patético -así los análisis sobre la personalidad y sobre los planteamientos teológicos de Lutero- el catolicismo, por su parte, parecería racionalizar, y equilibrar, esa dimensión existencial allí expresada patéticamente en términos de una actitud ética que le confiere a las dimensiones cognitivas un más fuerte papel. Habria, asi, un «talante protestante» frente a un «talante católicon y la hipótesis rezaría que la distinción entre ambos talantes pudiera explicar multitud de otras diferencias entre las respectivas maneras de habérselas con el mundo. Como luego explicaría Aranguren en el Prólogo de 1980, en unas magníficas páginas de autocrítica o de autoexplicación, el libro arrancaba con la contraposición entre las formas del catolicismo de D'Ors y de Guardini, un catolicismo «liturgista, noético y lúdico-ritual», y las formas filosófico-existenciales, patéticas y trágicas del cristianismo existencial. En ese sentido, la idea de talante apuntaba a diferentes constelaciones de disposiciones anímicas, la de un «talante bien temperado y esperanzado" católico y la de un «talante angustiado y desesperado (Lutero, Pascal, y hasta cierto punto, Kierkegaard y Unamuno)». El orteguismo del Aranguren de aquellos años (un orteguismo

\footnotetext{
${ }^{2}$ Obras Completas, vol. I, p. 211.
} 
que cederá el lugar de primacía o de evidencia a la matriz filosófica zubiriana en la Ética de seis años después ${ }^{3}$ ) hacía que esa idea de talante, o de diversidad de talantes, jugara también en relación con otra de orden más sociológico o cultural, la de «situación». Si la idea de talante apuntaba a una posiblc tipología de las disposiciones anímicas, sobre bases problemáticamente psicológicas, la de situación intentaba, por su parte, apuntar al análisis de los contextos sociales, históricos y culturales en los que los hombres viven y configuran sus creencias. Pues bien, el libro, quizá sobre todo en su análisis de Lutero, parece operar rígidamente sobre esas contraposiciones entre los dos talantes y de éstos, a su vez, con la jea de la situación histórica. Pero pronto empieza a percibirse que esa tensión entre el talante (páthos) y la actitud (êthos) -tal como aparecerán ya claramente diferenciados en la Ética seis años después en un contexto teórico distinto- no podría ser aplicada con resultados explicativos adecuados a una contraposición entre protestantismo (patético) y catolicismo (ético); eso, parece percibir pronto Aranguren, sería cometer una suerte de falacia de mala aplicación categorial. En efecto, en su autoexplicación de 1980 Aranguren señala que pronto entendió que esas contraposiciones -entre formas de talante y entre éstas y las situacionesno se sostenían. Como ya se indicaba al final del libro, «el talante no es por sí un factum primariamente independiente e irreductible, sino que se halla siempre en función de una situación» ${ }^{4} \mathrm{y}$, por lo tanto, y matizando to que antes se nos había sugerido, indica ahora que no podemos comprender a Lutero sino añadiendo a su disposición anímica los efectos de una honda crisis histórica, de una situación. Al final de Catolicismo y protestantismo..., pucs, se alza acta de la imposibilidad de mantener la distinción antropológica entre protestantes y católicos y, consiguientemente, la hipótesis inicialmente formulada: si la idea de talante puede mantenerse $-y$ puede haber buenas arangurenianas razones para hacerlo, como veremos en seguida - habrá de ser en otro contexto, con otros fines.

No es objeto de estas líneas el trazar, ni siquiera mínimamente, un apunte de la evolución teórica de Aranguren. No obstante, cabría indicar que Catolicismo y protestantismo... se ubica en una peculiar y cambiante encrucijada en ese desarrollo filosófico. Parte de D'Ors (y, también, de Ortega) pero se encamina hacia el existencialismo al que lee, quizá, más desde Ortega que de un D'Ors con el que marca crecientes distancias; arranca del catolicismo pero se encamina hacia su existencialización y, según el diagnóstico cultural y teológico que el libro mismo presenta, hacia su «protestantización». Es importante indicar - a efectos no sólo de la comprensión del momento de Aranguren, sino de las alternativas que se le ofrecían a la filosofía española en aquel

* No se olvide, no obstante, que la defensa de Ortega frente al P. Ramírez es estrictamente coetánea de la Ética, en 1958. No hay abandono de Ortega, pues, sino-como veremos en seguidael empleo de otro utillaje para la elaboración de la idea de talante.

$O C, 1$, p. 386. 
momento- la vía media que se afana en trazar: «Ni Unamuno, ni D'Ors» ${ }^{5}$, a pesar de las respectivas cercanías o distancias con respecto a los dos. EI horizonte tenso entre Ortega y Zubiri que marcaba al Aranguren del final de los cincuenta y los sesenta estaba ya expedito desde el comienzo de aquella primera década. El conjunto de influencias teóricas -y a las mencionadas hay que añadir la filosofía existencialista de preguerra, como indicaremosvan ponderándose y reequilibrándose, como una constelación en movimiento. Pero, en ese contexto, el existencialismo que incluso en 1980 se reclama como clima del libro (y como recuperación, por lo tanto, de lo que de válido y de necesario hubiera en la idea de talante) queda marcado de una forma peculiar: por el interés sociológico y cultural en las formas de vida que se alentaba tras Ortega y por el interés por la estructura racional de nuestra manera de ajustarnos al mundo (nuestra segunda naturaleza) que abría Zubiri. Menos Heidegger de lo que, incluso, su presencia recurrente en este libro indica; nada, por ejemplo, de la recuperación francesa del autor de Sein und Zeit en aquellos años. Las repetidas referencias a Heidegger son, quizá, significativas desde las (todavía no explícitas) categorias zubirianas y cl uso, tanto en 1952 como en 1958, de sendas nociones heideggerianas (cuales son Befindlichkeit y Geworfenheit) es, por así decirlo, ancilar con respecto a los propios intereses zubirianos de Aranguren. El existencialismo arangureniano, si es que de él puede hablarse, es más epocal que estructural en sus reflexiones. Si el mencionado interés sociológico y cultural requerirá, en momentos sucesivos, de nuevas categorías y de otros marcos de análisis (en Catolicismo y protestantismo..., por ejemplo, sólo aparece una vez, marginalmente, Weber) y habrá de incorporar, por lo tanto, otros lenguajes a la matriz orteguiana, el análisis de la acción moral, la ética docens, permanecerá más fielmente heredero de Zubiri.

En este contexto el concepto de talante juega un especial papel: se proyecta como la categoría mediadora entre el análisis de una forma de existencia o una manera de ser, por una parte, y una circunstancia histórica, una situación, por otra; pero esa proyección puede entenderse, a su vez, más bien existencialmente o más bien como instrumento para la comprensión del hecho cultural de la moralidad. Si atendemos a lo que en el libro se lee y a lo que Aranguren señaló años después sobre Catolicismo y protestantismo..., el talante comienza allí por ser entendido de una manera que se escora hacia los primeros términos de esa proyección: es una categoría centrada más bien en el análisis existencial de una forma de existencia. Hacia al final, no obstante, se descubre que la categoría mediadora que es el talante habrá de incorporar más ponderadamente los segundos términos de la proyección: la situación y la ubicación más estructural que psicológica del individuo en su situación. Asistimos, pues, a una cierta "des-psicologización» de la idea de talante. En efecto, aunque Aranguren señala específicamente que «talante» traduce «Stimmung» en tanto disposición

\footnotetext{
${ }^{3} O C, 1$, p. 213 .
} 
anímica, entendida en un contexto de psicología existencial, y aunque se lamente, incluso, con Heidegger, del poco desarrollo de esa noción ${ }^{6}$, cl uso más lato que de esa noción se hace indica ya más bien en dirección distinta. Desde luego, no hacia la interpretación de la Stimmung que podría hacer Kant: la filosofia moral kantiana, en sus aspectos estructurales y conceptuales, no está en el primer plano de la ética arangureniana.

Aranguren no reclamaba, en los primeros años cincuenta, la originalidad de la idea de talante, aunque indicara su falta de sistematización anterior. Tenemos que esperar a la nueva presentación de su teoría en El buen talante para que, reteniéndose no obstante las refcrencias a la Befindlichkeit heideggeriana, traducida como "temple fundamental", se indique que «es curioso observar que los pensadores españoles, aun los más afines a la filosofía de la existencia, (...) apenas han parado mientes - excepto Unamuno- en el estado de ánimo o talantes ${ }^{7}$. Ni Zubiri - a quien se le exculpa por la forma abstracta e intelectualista de su pensamiento- ni Ortega - para cuya falta de atención al análisis de este tipo de disposiciones encuentra Aranguren menos comprensibles razones- pudieron servirle, parece decírsenos, en la elaboración de esa noción. Nosotros, a pesar de las referencias, hemos señalado que tampoco Heidegger. Es, pues, una categoría específicamente propia la que juega en esas complejas y movedizas constelaciones conceptuales a las que nos hemos referido.

¿Cuál es el contenido de esta compleja noción? Podemos proponer que en Catolicismo y protestantismo... el talante se define en tres ejes que no desaparecerán posteriormente, a pesar de ciertas alteraciones: desde un acercamiento a «los estados de ánimo» fundamentales, o estructurales, del individuo; desde los efectos de tales estados en la definición del punto de vista, o mejor, de la forma de la acción, de ese individuo en su contexto cultural y social; por último, y señaladamente, en la definición de la actitud religiosa básica o radical.

El talante, en primer lugar, es una manera de encontrarse. «El hombre, cada hombre, se encuentra siempre en un estado de ánimo (...) [que] condiciona y colorea nuestro mundo de percepciones, pensamientos y sentimientos» ${ }^{8}$. Con estas palabras comienza la Introducción a Catolicismo y protestantismo... Más adelante la metáfora del color se precisa y con ella se da entrada al segundo eje, a la constitución de la mirada sobre el mundo: «Nuestra cambiante luz interior ilumina unas veces ésta, otras aquella cara del mundo; la realidad se nos aparece así como un reflejo del mundo" ". Dos polos parecen aparecer en esta primera dimensión, la manera de encontrarse, que es el talante. No

${ }^{6}$ Este lamento compartido con Heidegger le parece menos ajustado a Aranguren en los años posteriores a la aparición de Sein und Zeit, pero lo sostiene en lo que a la ética se refiere. Cfr. Ética, en $O C$, II, p. 393.

$O C$, II, p. 631.

${ }^{*} O C, 1, \mathrm{p}, 217$.

${ }^{9}$ Ibid. 
parece que el talante sea un sentimiento (no es, pues, una tesis emotivista la que comporta); pero implica una cierta movilidad que determina, como veremos en seguida, un mundo. El talante parece, pues, referir a la manera de mirar, a la estructura anímica del mirar, con la que nos enfrentamos y definimos el mundo. $Y$ "anímico» parece indicar no un estado pasajero (como un sentimiento o una percepción, en sí cambiantes), sino una cierta constancia de cómo somos. Más adelante apuntará Aranguren una idea que, años despućs, precisará en El buen talante: existe una «jerarquía de estados de ánimo» (en concreto, una «jerarquía gnoseológica de estados de ánimo») ${ }^{10}$. en la raíz, la forma de disposición que se nos requiere cuando nos cuestionamos la verdad de algo; en la superficie, deducimos, la más coloreante movilidad de nuestro ánimo alterable. La verdad, la actitud hacia la verdad, no es sólo epistémica o cognitiva; refiere a una forma de ser frente al mundo (así la modalidad griega, teórica, en cuyas interpretaciones heideggerianas se detiene -críticamente- Aranguren ${ }^{11}$ ). Pero esta fundamental manera de ser ante el mundo no es, quizá, la última. La estructura gnoseológica depende, veremos que de peculiar manera, de una estructura ética; en un texto suprimido en la reescritura del texto más de treinta años después ${ }^{12}$ se señala: «No hay (...) un único estado de ánimo apto para el conocimiento. Pero hay, sí, una jerarquía de estados de ánimo y en lo alto de ella están - buen talante - la csperanza, la confianza, la fe, la paz» ${ }^{13}$. Que el buen talante no pueda definirse años después con esa descripción de virtudes teologales no niega, no obstante, que no se retenga esa prioridad ética (de las virtudes éticas sobre las dianoéticas) a la hora de entender la idea de talante.

No obstante, definir esa última raíz caracterial (ética) del talante debe conducir a precisar qué entendemos por carácter. Aranguren lo hace oponiendo, con Bollnow, talante a actitud. El talante es, por así decirlo, el lado «dado» de nuestro carácter ético; la actitud seria su cara «construida», por el trabajo de nosotros sobre nosotros mismos que podemos denominar, adecuadamente, la construcción moral de la personalidad. Aunque años después se abandonará, de nucvo, la peculiar descripción connotada en esá contraposición entre talante y actitud y se modificará, por endc, la definición misma de talante, se conserva la definición cuasi-d'orsiana con la que en Catolicismo y protestantismo... se indicaba: «El sentimiento, estado de ánimo o talante (y nótese que anteriormente no se identificaba sentimiento y talante, lo que es ejemplo de los desplazamientos semánticos del término en esos años, C. T.) es una disposición espontánea, pre-racional (...). El hombre adánico (...) cs cl más propicio a

5éase $O C, 1,220$, y $O C, 11,632$. Éste es un buen ejemplo de la reescritura de esta úlima obra. En ella, excepto en las tres páginas finales, se mantiene el texto anterior, pero éste está estruclurado de manera diversa, con alteraciones de orden, etc.

$" O C$, II, pp. 632 yss.

12 Aprovechemos para indicar que, si hay un rasgo común en lo suprimido, es aquello que hacia de Catolicismo y protestantismo... un texto utens en la definición del talante católico.

oc, $1, \mathrm{p}, 225$. 
entregarse a sus estados de ánimo. Por el contrario, el apoyo en una tradición, la seguridad, el descanso en una fe racionalmente justificada, la posesión de una firme concepción de la vida, convierten el «talante» en «actitud», dan «sentido» a la vida, le prestan «estilo». La actitud es, pues, un talante no ya desnudo, sino informado y ordenado, penetrado de logos» ${ }^{14}$. Creo que lo que luego se acaba abandonando (y no aparece ya en Ética) es la connotación demasiado irracional de lo que aquí se llama pre-racional (y luego se llamará pre-moral), a la vez que lo racional (lo ético) no aparece ya como descanso, firme asentamiento, etc. Pero el doble aspecto de lo «dado» y lo «construido» moralmente se mantiene. Lo pre-racional, el talante, será páthos, el ámbito de lo pre-moral.

El tercer y último eje de la idea de talante en Catolicismo... es, quizá, el más importante para el Aranguren de aquellos años y para el análisis del cual pareció elaborarse la idea misma de talante: la dimensión religiosa. Para Aranguren existe una fundamental «configuración religiosa del modo personal de ser» ${ }^{15}$, una idea que, en aquel libro, se elevaba junto al supuesto de que «la religión es el núcleo central de la filosofía y, en general, de la cultura todas " ${ }^{6}$. Ya indicamos que el inicial problema que el libro se proponía tratar era la diferencia entre los talantes protestante y católico. Dado tal objetivo no son de extrañar las frases que acaban de citarse. Pero también sabemos, por la conclusión de ese mismo libro y por su Prólogo de 1980, que tal objetivo no se alcanzó. En efecto, «el talante no es un factum primariamente independiente e irreductible, sino que se halla siempre en función de una situación» ${ }^{17}$, se nos dice en ese final para modificar la hipótesis de arranque, pues «también puede ser dramática la existencia religiosa del hombre católico» y no sólo el protestante, fruto de su patético talante ${ }^{\mathrm{is}}$. La conciencia de que "estamos atravesando una "noche oscura" " $"$ hace ya inevitable tal dramatismo, tal «existencialización», con la que, como se nos ha ido argumentado en las páginas anteriores, estarían en plena sintonía las formas protestantes del talante y las formas existencialistas del catolicismo.

¿Cómo queda la idea de talante tras esta crítica? La noción contenía otros elementos junto al papel que se le quería hacer jugar en la hipótesis rcchazada, un rechazo que tiene, por su parte, la virtud de modificar y enriquecer aquella noción misma, enlazándola con un distinto enfoque en la comprensión de las formas culturales y las situaciones históricas. Los otros elementos - que podrán ser recuperados en el distinto lenguaje teórico de la Ética-son precisamente los que apuntan al nexo entre la manera de encontrarse en el mundo y la manera ética de hacerse. A ellos nos volvemos, pues, en el texto de 1958.

\footnotetext{
${ }^{14} O C, 1, p .225$

is $O C, \mathbf{1}, \mathrm{p} .230$.

16 Ibid.

${ }^{17} O C, 1, \mathrm{p}, 386$.

* $O C, \mathrm{I}, \mathrm{p}, 390$.

OC, I, p. 399 .
} 


\section{El talante como páthos}

El capítulo once de la segunda parte de la Ética se denomina «El talante». Introduce, junto con el que le antecede, el tratamiento de las virtudes y muestra con relativa claridad lo que hubiera de escolástico y de aristotélico en el pensamiento de Aranguren. Esta veta aristotélica pudiera ser lo que hace más actual el concepto, al menos si lo relacionamos con la continuada recuperación de la idea de sensibilidad moral por medio de la cual la ética analítica más reciente ha contrapesado los sesgos bien estrictamente cognitivistas o bien, por contra, radicalmente emotivistas de los planteamientos filosófico-morales de los años cincuenta y sesenta ${ }^{20}$. Desde estas nuevas lecturas, la virtud aristotélica es una manera de ser que refiere tanto a las pasiones (páthos) como a las acciones y es una manera de ser que se conforma y realiza de manera determinada. No podríamos, en sentido aristotélico estricto, entender qué sea la moralidad sin entender este trabajo y esta conformación de nuestra sensibilidad junto a la crítica o la iluminación de nuestras acciones. Sobre ello regresaremos, aunque sea brevemente, más adelante.

Aranguren cntiende ese páthos precisamente como esa peculiar forma de la sensibilidad estructural, por así decirlo, en el ser humano. Es «lo que se siente, el sentimiento fundamental, el modo de enfrentarse emocionalmente, es decir, por naturaleza, con la realidad, "el estado del alma", o lo que yo he solido llamar "talante" ${ }^{21}$ ". Como ya veíamos en el epígrafe anterior, «el talante nos es dado" y por ello entiende Aranguren que puede ser relacionado con la Geworfenheit heideggeriana (aquel estar arrojados que Gaos, torturando la lengua, tradujo como «estado de yecto»), aunque se aleje de lo que califica de irracionalismo del existencialista alemán. En esas páginas la matriz zubiriana del pensamiento arangureniano aparece con claridad: lo que nos viene dado no es la forma fundamental de nuestro relacionarnos con el mundo; lo es, más bien, «la inteligencia, entendida como estar en la realidad y como constitución de ese mundo que después el páthos va a colorear emocionalmente con una gama fría o encendida, con una paleta oscura o ardiente» ${ }^{22}$. Nuestra apertura a la realidad, aquel ajuste zubiriano a la realidad que tan importante papel jugará en otros momentos de la Ética en referencia a la «moral como estructura», se «tonaliza» (por emplear metáforas musicales, menos caras a Aranguren que las pictóricas) por las formas del pathos, del talante. Esta modulación tiene raíces biológicas: «lo que biológicamente aparece como "tono vital" (...) es, en cuanto anímicamente vivido, el talante» ${ }^{23}$. También, de manera

Nos referimos a autores cono M. Nussbaum, J, McDowell y D. Wiggins. Sobre estas nuevas teorias de la sensibilidad moral puede verse, entre otros, el trabajo de S. Darwall, A. Gibbard y P. Railton "Toward Fin de siècle Ethics», The Philosophical Review, 101, 1 (Jan. 1992).

"OC, II, p. 393.

"OC, II, p. 394.

${ }^{23}$ Ibid. 
no precisada por la psicología (cuyas aportaciones lee Aranguren zubirianamente), aparece en referencia a nuestra percepción unitaria de la realidad.

El talante, pues, aparece como páthos dado. Frente a él, modulando nuestro ajuste al mundo ( $c$ o siendo una forma de ese ajuste?), el êthos o el carácter hecho, un concepto en el que resuena aquella «actitud" de Catolicismo y protestantismo... Si «páthos» es, pues, lo dado en la naturaleza, el êthos habrá de ser, activamente configurada, «segunda naturaleza» ${ }^{24}$. "Talante y carácter son, pues, los dos polos de la vida ética, pre-moral el uno, auténticamente moral el otro" ${ }^{25}$. Quizá sea el momento de plantear algunas dudas de interpretación. Parecería, en primer lugar, que el talante, esa modulación de nuestro ajuste inteligente al mundo, nos viene dado. Parecería, por lo tanto, que su modificación nos es, en general, inalcanzable, y que lo que modificamos es nuestro carácter, no nuestro talante. Parecería, pues, que nuestro acoplamiento a la realidad cn cl orden de la sensibilidad no es, como la cita que acabamos de recordar indica, moral, sino pre-moral. Parecería, por último, que el rango moral de la idea de talante, en la que insistíamos anteriormente, queda fuertemente cuestionado.

Tal vez haya aquí una situación aporética. Por un lado, la relevancia, lo crucial, de la idea de talante para la ética; por otro, su carácter no ético, pre-moral. Pero cabría sugerir que esa aporía lo es, sobre todo, de lo que liamamos la matriz zubiriana del pensamiento de Aranguren, no de las intuiciones que él venía persiguiendo. La matriz zubiriana solventaría la cuestión, como hace la Ética, indicando la indisoluble unidad de talante y carácter, de ajuste y de su modulación, de sentimiento e inteligencia. Pero, desde esa percepción, no acabamos de saber si lo dado — que coloreaba nuestra actividad de ajuste a la realidad- es ello mismo susceptible de ser modificado, como un aristotelismo más claro exigiría, o si sería una constante estructural. (A no ser, como otras veces parece sugerirse, que hubiera dos niveles de talantes: uno estructural, inmodificable; otro más mutable y susceptible de conformación en el carâcter. Pero esa posible lectura queda, también, rechazada en otros textos.) Una definición de lo que el hombre es (en aquello que se llamó antropología) o de lo que la realidad es (en lo que se denominó metafísica) hace problemática, por muy dinámicas que ambas definiciones sean, la consideración de lo que el hombre y la realidad deberian ser, aspirarían a ser o desearían ser (sea cual sea la forma de modalidad que demos en adoptar para definir lo moral). Aunque Aranguren abra la ética en 1958 a la metafísica (y también a la religión), las relaciones entre ambas nunca han dejado de ser problemáticas a no ser que se entendieran ya en otro contexto.

Esta inestable situación queda claramente definida por Aranguren en los párrafos finales del capítulo sobre el talante de la Ética. Pero entre ellos aparece

${ }^{34}$ OC, II, p. 395 .

${ }^{25}$ Ibid. 
una idea nueva, casi insospechable: «El talante es - se nos dice ahora- la materia prima, el thymós o fuerza que posecmos para la forja del carácter ${ }^{26}$. La idca es, según lo que vamos viendo, sorprendente y ajustadamente aristotélica, y quiebra no pocas de las ideas hasta ahora scñaladas: la sensibilidad conforma la moralidad y es conformada en ella, motiva nuestros actos y opera como su condición de interpretación. $Y$ esa idea parece ir a contrapelo de aquello «dado», «inmodificable» que hace uno momento veíamos. Pero, quizá, nos ayudará en el último paso de nuestro análisis.

Hemos venido diciendo que las relativas oscilaciones semánticas de la palabra talante, en esos distintos contextos teóricos y usos, no parecen, no obstante, difuminar un cierto aire de familia de ese término, un aire al que Aranguren pareció ser fiel en diversos - y distintos - momentos té́ricos. Que los diversos marcos conceptuales - existencialista, cuasi-orteguiano, zubiriano- induzcan deslizamientos y aporías no quita para que percibamos una sintonía entre los intereses de Aranguren al acuñar y emplear diferenciadamente esa noción. Ese aire refiere, estimo, a lo que encontramos de la sensibilidad del ser humano a la hora de definir su manera de ser y es a lo que párrafos atrás apuntábamos como recuperación de Aristóteles en la filosofía contemporánea. Por retomar términos aristotélicos, la manera de ser - que no es una modalidad o categoría del ser- refiere, en primer lugar, a lo que es susceptible de ser hecho ser de manera distinta porque opera no el orden de la necesidad, sino de la contingencia. Por lo tanto, las maneras de ser son las que refieren más a nuestro hacernos que a cómo decimos conocer o ajustarnos - estructuralmente- con el mundo. Pues bien, ese hacernos no es, en términos aristotélicos, segregable (aunque sí analíticamente diferenciable) en una sensibilidad y una razón independientes: nuestra sensibilidad conforma, motiva, nuestro razonar práctico (junto a otras cosas, evidentemente) a la vez que puede ser modificada, estructurada, configurada por ese razonar (junto a otras cosas, evidentemente). Tal vez la tonalidad escolástica y zubiriana de la Ética (en matizada confluencia) haga posible la sintonía de Aranguren con estos análisis de la sensibilidad moral a los que hacemos referencia. Tal conclusión no deja de ser sorprendente, pero permitc una relectura de la idea de talante en términos renovados (por muy clásicos y rancios que sean), quizá conceptualmente más precisos en sus usos y efectos.

Aunque no podamos ahora precisar ese programa de relectura, valgan estas notas para sugerirlo. En efecto, el carácter estructural del talante (en tanto disposición anímica) se corresponde con el interés de obviar el insoluble dilema de la ética contemporánea entre cognitivismo y emotivismo, o entre racionalidad práctica y sensibilidad. Si el talante es categoría puente de esos niveles (o elementos) de nuestra acción y ser morales, es decir, si el talante no es sólo pre-moral, sino tonalidad de lo moral, dicho puente permitiria pensar que el «ajuste» zubiriano con el mundo lo es también de una sensibilidad que

\footnotetext{
${ }^{25} O C_{\mathrm{s}}$ II, p. 396.
} 
no tiene carácter ancilar con respecto al conocimiento sino que conforma, también, ese ajuste.

A ello cabe añadir un rasgo, aparentemente secundario pero crucial, sobre el que Aranguren insistió en su primera presentación de la noción de talante, al que fue fiel en otros momentos posteriores y que ha reaparecido con fuerza en las discusiones morales posteriores: la relación de nuestro talante (conformador de nuestro mundo y conformado por nuestra acción en el mundo) con las prácticas retóricas, literarias. Aunque Aranguren insistió, en 1952, en cómo nuestra manera de ser determina nuestra manera de ver y de relatar el mundo, también señaló cómo determinadas lecturas nos sumergen en el talante que en ellas se refleja. La «participación en el estado de ánimo ajeno [es] en lo que consiste la comunicación espiritual, la "simpatía", el entendimiento profundo entre los hombres» ${ }^{27}$, nos dice comentando el efecto de la lectura de textos literarios. Es decir, el entendimiento no lo es sólo en el orden de las razones (en rigor aristotélico y arangureniano tal no sería posible), sino en el orden de aquellos hechos lingüísticos (textos, hablas) en los que se configuran y plasman talantes e ideas, en los que se construyen talantes e ideas.

Por último, el nexo que se establece entre talante y situación permite entender que la acción humana, y en concreto la acción moral, remite a estructuras de interacción históricamente moduladas, una idea a la que no ha podido tampoco ser ajena la filosofía moral contemporánea, esta vez en las formulaciones dialógicas y comunicativas de la teoría de la sociedad. Los intereses del Aranguren moralista - atento a las formas emergentes de moral e incitador de las mismas - de años posteriores a los textos que hemos comentado se centraron en el análisis de las formas sociales de la moralidad. En esos análisis la idea de talante aparece en contextos no tan teóricos como los que hemos venido señalando, pero retiene parte del hálito con el que aparecía en Catolicismo y protestantismo... y con-suena con estos nuevos análisis de las formas sociales de construcción de la subjetividad.

Tal vez lo dicho pueda hacer, por lo tanto, plausible la idea que sugeriamos al principio de estos párrafos: que la idea de talante traduce (y anticipa) en buen castellano algunos temas de las discusiones presentes. Desligar esa idea de sus contextos de interpretación para retener su trasfondo no traiciona, por otra parte, a un autor nada escolástico de sí mismo.

\footnotetext{
II $O C, \mathrm{I}, \mathrm{p} .222$.
} 